\title{
Endoscopic closure of gastrocolocutaneous fistula following percutaneous endoscopic gastrostomy, by OverStitch Endoscopic Suturing System
}

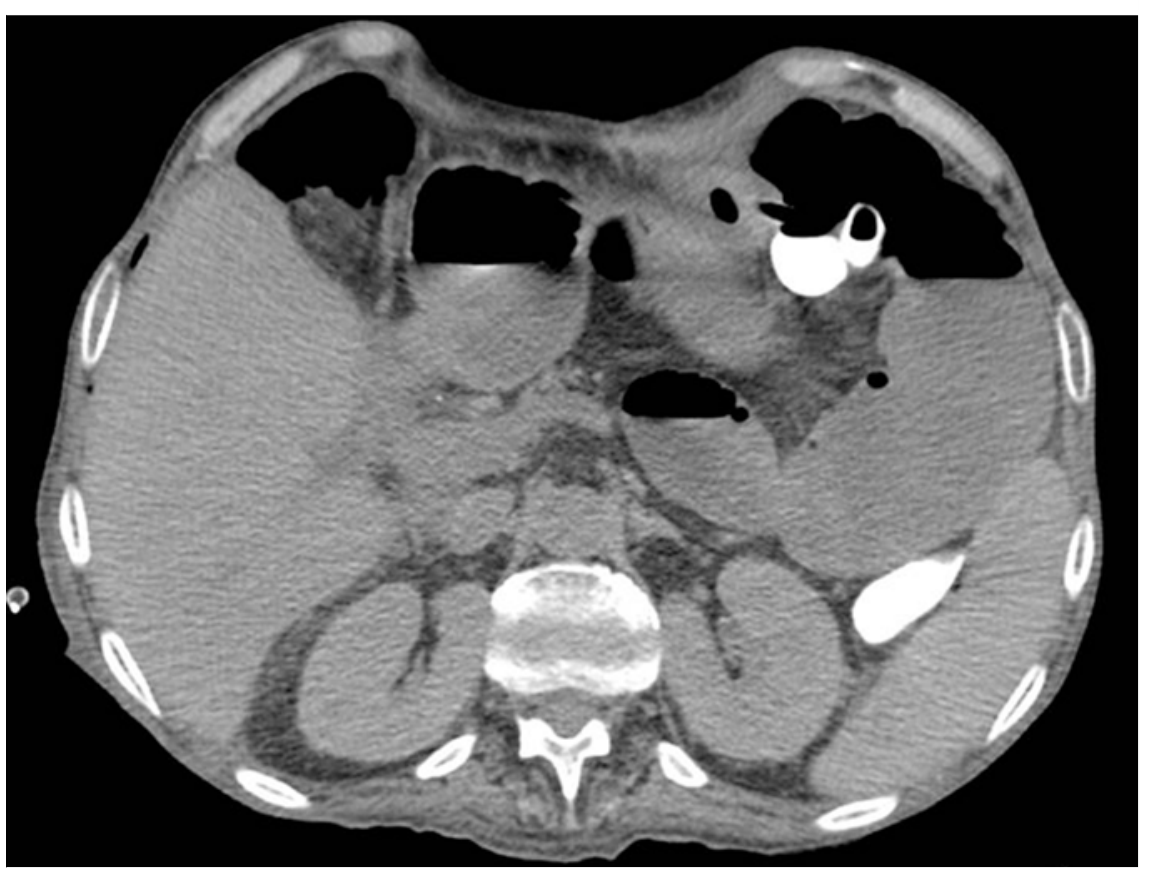

- Fig. 1 Contrast-enhanced (Gastrografin) computed tomography scan. Percutaneous endoscopic gastrostomy (PEG) device migrated into the transverse colon, with bowel opacification and without extraluminal spreading of the contrast medium.

A 48-year-old liver transplant patient with acute graft rejection, sepsis, and malnutrition was referred to our unit for a percutaneous endoscopic gastrostomy (PEG).

The PEG procedure was uneventful, and a correct positioning of the bumper in the stomach was documented. Three days after the procedure, vomiting and abdominal distension occurred. A computed tomography (CT) scan with hydrosoluble contrast agent showed dislocation of the bumper into the transverse colon, with bowel opacification, due to the presence of a gastrocolic fistula. No leak of contrast into the peritoneal cavity was detected ( $\triangleright$ Fig. $\mathbf{1}$ ).

Endoscopy showed a transmural defect of $2 \mathrm{~cm}$ in diameter in the anterior wall of the gastric body ( $\mathbf{F i g . 2}$ ). To close the fistula, we first used forceps to scar the mucosal margins in order to promote cicatrization. We then approximated the opposite margins of the wall defect by placing three simple sutures using the OverStitch Endoscopic Suturing System (Apollo Endosurgery, Austin, Texas, USA) ( $\triangleright$ Video 1). An intraprocedural contrast radiograph showed no colonic opacification.

A same-session colonoscopy revealed, in the transverse colon, the bumper ( $\triangleright$ Fig.3a,b), which was grasped and retrieved from the colon following sectioning of the extracorporeal PEG tube. The fistula was then closed with clips ( $\vee$ Fig. $3 \mathbf{c}$ ). At the end of the procedure, contrast radiography found no leak of

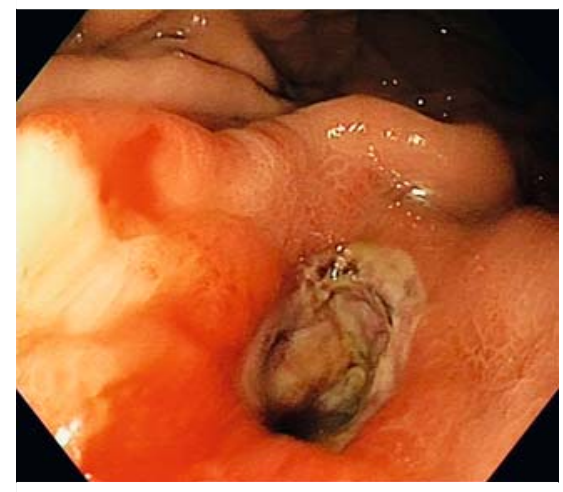

- Fig. 2 Upper gastrointestinal endoscopy showing a transmural defect of the anterior wall of the gastric body, covered with fibrin.

contrast from the colonic side of the fistula; the cutaneous side of the fistula healed by secondary intention. An endoscopic control after 10 days showed both gastric and colonic aspects of the fistula correctly repaired. Regular enteral feeding through nasogastric tube was resumed.

A gastrocolic fistula is a rare complication of PEG and may require surgery [1]. Endoscopic management with clips has been described [2,3], even if it may not be feasible in the event of a large fibrotic wall defect. In our case, we found that the OverStitch Endoscopic Suturing System is a feasible and effective treatment tool for a large gastrocolocutaneous fistula.

Endoscopy_UCTN_Code_CPL_1AH_2AI

Competing interests

None 

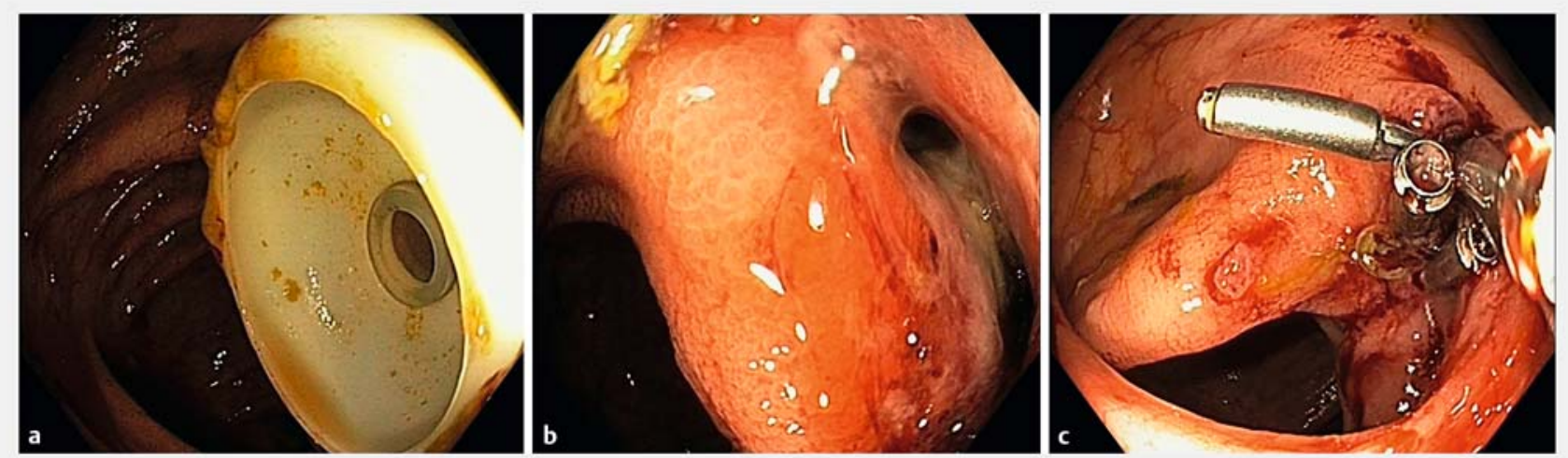

- Fig. 3 Colonoscopy. a PEG bumper migrated into the transverse colon; b colonic wall defect following removal of the bumper; c endoscopic closure of the colonic aspect of the gastrocolic fistula with clips.

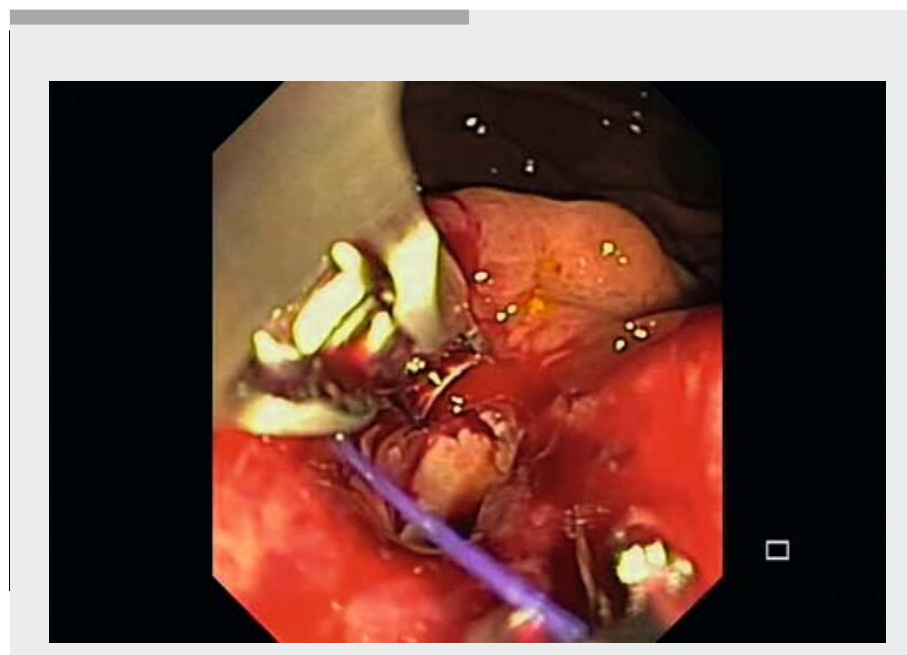

Video 1 Video showing endoscopic closure of the gastric aspect of the gastrocolic fistula using the OverStitch Endoscopic Suturing System.

\section{References}

[1] Schrag SP, Sharma R, Jaik NP et al. Complications related to percutaneous endoscopic gastrostomy (PEG) tubes. A comprehensive clinical review. Gastrointestin Liver Dis 2007; 16: 407-418

[2] Bertolini R, Meyenberger C, Sulz M. C. first report of colonoscopic closure of a gastrocolocutaneous PEG migration with over-thescope-clip-system. World J Gastroenterol 2014; 20: 11439 - 11442

[3] Lee J, Kim J, Kim H et al. Gastrocolocutaneous fistula: an unusual case of gastrostomy tube malfunction with diarrhea. Clin Endosc 2018; 51: $196-200$

\section{Bibliography}

DOI https://doi.org/10.1055/a-0956-6792

Published online: 5.7.2019

Endoscopy 2019; 51: E384-E385

(c) Georg Thieme Verlag KG

Stuttgart · New York

ISSN 0013-726X

\section{ENDOSCOPY E-VIDEOS}

https://eref.thieme.de/e-videos

Diagnostic and Therapeutic Services, IRCCS ISMETT - UPMC, Via E. Tricomi 5, 90127

Palermo, Italy

Fax: +390912192400

dligresti@ismett.edu
Trapianti e Terapie ad alta specializzazione), Palermo, Italy

2 Hepatology Unit, Department for the Treatment and Study of Abdominal Diseases and Abdominal Transplantation, IRCCS ISMETT (Istituto Mediterraneo per i Trapianti e Terapie ad alta specializzazione), Palermo, Italy

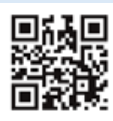

Endoscopy E-Videos is a free access online section, reporting on interesting cases and new techniques in gastroenterological endoscopy. All papers include a high quality video and all contributions are freely accessible online.

This section has its own submission website at

https://mc.manuscriptcentral.com/e-videos 